P196 (continued)

Objective: The objective of this study was to assess the feasibility of using digital template photos to estimate children's dietary intake in childcare settings.

Study Design, Setting, Participants, Intervention: Photos of preschool children's meals were taken before consumption, when any additional servings were provided, and after consumption. Weights and template photos of each meal component were taken separately, at decreasing portion sizes beginning with the serving size given during mealtime and ending at approximately onequarter to one-eighth of the original serving size, such that if any more were eaten, it would be considered finished. Three trained undergraduate research assistant coders compared photos of actual mealtime plates with the digital template photos side by side on a split screen. Coders used the digital template photos to visually estimate the portion size of each meal component as it had been served and consumed.

Outcome Measures and Analysis: Preliminary data from ongoing data collection consisting of 10 mealtimes composed of 35 food and beverage components in total were independently coded. Inter-rater reliability was evaluated by intra-class correlation coefficient (ICC).

Results: Across the 10 preliminary trials, ICC was 0.931 suggesting trained coders can reliably estimate food quantities using digital template photos.

Conclusions and Implications: As a feasible and costeffective method, digital template photos may create opportunities for assessing children's dietary intake in research and intervention projects where it has been otherwise prohibitive to do so.

Funding: USDA.

\section{P197 Food and Nutrition Literacy Research: Content Analysis}

Nazan Aktas,PhD, naktas@selcuk.edu.tr, Selcuk University, Faculty of Health Sciences, Department of Nutrition and Dietetics, Ardiçli District, Ismetpasa Street, Selçuklu, Konya, 42250, Turkey; Yahya Ozdogan, PhD, Ankara Yıldırım Beyazıt University, Faculty of Health Sciences, Department of Nutrition and Dietetics; Hilal Hizli Güldemir, MS, Istanbul Medipol University, Faculty of Health Sciences, Department of Nutrition and Dietetics; Sahika Turken

Background (Background, Rationale, Prior Research, and/or Theory): The term food and nutrition literacy, which is commonly used in the literature in recent years, involves accurate knowledge, attitude, skill and behaviors about food, nutrition, food choice, sustainable nutrition, cooking, food safety and healthy eating.

Objective: The aim of this study is to conduct content analysis of the articles about food and nutrition literacy published in scientific journals between the years 2000 and 2017.

Study Design, Setting, Participants, Intervention: The articles whose full-text could be reached by entering the search words "food and nutrition literacy" on elec- tronic databases were examined with descriptive content analysis method using "the Food and Nutrition Literacy Paper Classification Form (FNLPCF)" developed by the researchers. The form includes information about the year of the study, the country, topics, key words, research design, theoretical foundation, study group, sample size, data collection tools, and analysis methods. The articles were also examined in terms of the definition of food and nutrition literacy and measurement tools.

Results: It was determined that studies on food and nutrition literacy increased quantitatively. Studies adopted a variety of research designs: quantitative, qualitative and mixed methods. Although the study groups in these studies included a variety of age groups, studies were mostly carried out with adult participants. Besides, it was ascertained that there was not a widely used measurement tool to measure food and nutrition literacy, but there were some studies to develop scales. It was also found that there was not a commonly agreed upon definition of the term "food and nutrition literacy."

Conclusions and Implications: It is highly crucial that food and nutrition literacy be examined scientifically for developing and sustaining healthy nutrition in the society. Moreover, it was concluded that together with academic circles, public and private sector organizations should cooperate to carry out more comprehensive and detailed studies to determine essential requirements of being food and nutrition literate, agreed-upon definition, and measurement methods.

Funding: None.

\section{P198 Poor General Health is Associated with Higher Percent Body Fat and Lower Appearance Satisfaction in African American Women}

Michika Nickerson, MPH, BS, Ohio State University, Medical Dietetics, School of Health and Rehabilitation Sciences, College of Medicine; Irene Hatsu, PhD, RDN, Ohio State University, Human Sciences, College of Education and Human Ecology; James Odei, PhD, Ohio State University, Department of Biostatistics, College of Public Health; Tracy Tylka, PhD, Ohio State University, Department of Psychology, College of Arts and Sciences; Wilson Figueroa, PhD, Ohio State University, Department of Extension and Medical Dietetics; Ingrid Richards Adams, PhD, LD, RDN, LDE, Richards.3@osu.edu, Ohio State University, Department of Extension and Medical Dietetics, School of Health and Rehabilitation Sciences, College of Medicine, $453 \mathrm{~W}$ 10th Avenue, Columbus, OH 43210

Background (Background, Rationale, Prior Research, and/or Theory): African American women (AAW) are at highest risk for obesity, putting them at high risk for obesity-related chronic diseases. Appearance dissatisfaction is a motivator for engagement in weight-loss strategies. On the contrary, AAW consistently demonstrate

Continued on page S102 
P198 (continued)

high levels of appearance satisfaction, which may keep them from engaging in health behaviors.

Objective: To describe the association between appearance satisfaction (AS), dietary habits, measures of adiposity and general health perception among AAW.

Study Design, Setting, Participants, Intervention: A cross sectional study of AAW adults in the southeastern U.S. $(n=74)$ recruited through flyers and snowballing techniques.

Outcome Measures and Analysis: Independent variables included adiposity (waist-to-hip ratio (WHR), percent body fat (PBF), fruit and vegetable intake and general health perception. AS was the dependent variable. The REACH 2010 St. Louis Healthy Heart Survey and Multidimensional Body-Self Relations Questionnaire-Appearance Scales (MBSRQ-AS) assessed health behaviors and AS. Pearson correlations were used to examine associations between variables of interest. Variables significantly associated with AS were placed into an ordinal regression models.

Results: Mean $\mathrm{PBF}=38.6 \%$, mean $\mathrm{WHR}=0.85 \mathrm{~cm}$, and mean AS score $=3.53$ (range $1-5)$. Mean fruit intake $=2.30$ and mean vegetable intake $=2.05$ servings per day. PBF was negatively associated with AS $(P<.001)$, [OR $=.93795 \%$ CI $(.892, .984)]$. Poor general health perception was negatively associated with AS $(P=.01),[\mathrm{OR}=.026,95 \% \mathrm{CI}$ $(.001, .449)]$.

Conclusions and Implications: Participants' appearance satisfaction increased as percentage of body fat and poor general health perception decreased. Appearance satisfaction was not associated with fruit and vegetable consumption. In general, AAW's appearance satisfaction is not a deterrent to their health. Despite associating poor general health perception and increased PBF with decreased AS, AAW in this sample did not engage in positive health behaviors. Practitioners should consider using metabolic indicators of health to motivate AAW towards positive health behaviors.

Funding: African American Studies and Research Program.

\section{P199 “Should We Go Out for Dinner Tonight?" Understanding Restaurant-Related Food Choices in the Family \\ Mackenzie Ferrante, MS, RD, Colorado State University; Rebecca Robbins, MS, RDN; Jeffrey Miller, PhD; \\ Gina S. Mohr, PhD; Laura L. Bellows, PhD, MPH, RD, laura.bellows@colostate.edu, Colorado State University, Department FSHN, 1571 Campus Delivery, Fort Collins, CO 80523-1571}

Background (Background, Rationale, Prior Research, and/or Theory): Families are dining out more than ever which may be problematic as eating out is associated with poor dietary quality. Understanding parent decisions related to dining out is important.

Objective: To explore factors influencing families' decisions related to eating out, and their practices and food choices in the restaurant setting.
Study Design, Setting, Participants, Intervention: A descriptive, cross-sectional study involving the design and dissemination of a survey for parents and caregivers with at least one child aged 4-12. The survey was developed using a compilation of peer-reviewed literature, five constructs from Furst et al.'s conceptual model of the food choice process, and exploratory interviews $(n=7)$. Once the survey was complete and put into an online platform, test-retest $(n=15)$ and cognitive interviews $(\mathrm{n}=3)$ were executed to assess validity and reliability. Convenience sampling was employed using email and social media to recruit participants.

Outcome Measures and Analysis: Results for testretest reliability were assessed using Spearman's rho. Descriptive statistics were used to examine survey responses and Cronbach alpha was used to test internal consistency of the theoretical constructs.

Results: Questions with weak reliability $(<0.6)$ were removed or changed during cognitive interviews $(n=15)$. Survey respondents $(n=349)$ were predominantly middleto high-income $(86.9 \%)$ mothers $(84.5 \%)$. Internal consistency was strong for Ideals $(\propto=0.79)$, Resources $(\propto=0.79)$, and Social Framework $(\propto=0.75)$. Food Context $(\propto=0.67)$ demonstrated moderate internal consistency, and Personal Factors $(\propto=0.52)$ demonstrated low internal consistency. Time, convenience, health, and the experience were the most important factors in the decision to eat out. Parents (63.6\%) limit children's food options, and 66.8\% stated children order from the children's menu.

Conclusions and Implications: This study provides a valid and reliable method, with strong theoretical framework, to assess family food choices when dining out. Findings provide insights into key influences of family decisions related to dining out and menus. Future research should explore strategies to promote healthful children's menu options complimenting parents' desired experience.

Funding: None.

\section{P200 SNAP-Ed Evaluation Framework: Southeast Regional Common Indicator Results} Celeste Doerr, PhD, Public Health Institute; Amy DeLisio, MPH, RDN, amy.delisio@wellness.phi.org, Public Health Institute, 1825 Bell Sreet; Suite 102, Sacramento, CA 95815; Suzanne Ryan Ibarra, PhD, MPH

Objective: To share an effective process and program results of an eight-state regional evaluation project that collected common indicators for policy, systems and environmental change (PSE) interventions.

Target Audience: SNAP-Ed practitioners and partners, nutrition educators, program evaluators, collaborative groups and coalitions.

Theory, Prior Research, Rationale: The Public Health Institute (PHI) Center for Wellness and Nutrition (CWN) conducted a series of collaborative calls with the Southeast SNAP-Ed state and local agencies to select common indicators from the SNAP-Ed Evaluation Framework. CWN conducted an analysis of current survey tools and

Continued on page 5103 